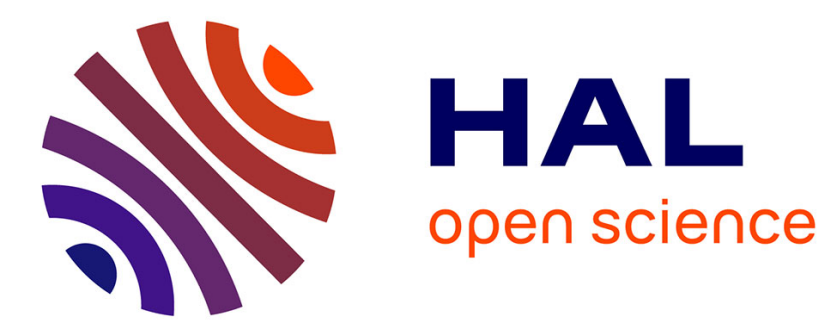

\title{
Modelling the dynamics of the piano action: is apparent success real?
}

\author{
Anders Thorin, Xavier Boutillon, José Lozada
}

\section{To cite this version:}

Anders Thorin, Xavier Boutillon, José Lozada. Modelling the dynamics of the piano action: is apparent success real?. Acta Acustica united with Acustica, 2014, pp.10. 10.3813/AAA.918795 . hal01193708

\section{HAL Id: hal-01193708 \\ https://hal.science/hal-01193708}

Submitted on 4 Sep 2015

HAL is a multi-disciplinary open access archive for the deposit and dissemination of scientific research documents, whether they are published or not. The documents may come from teaching and research institutions in France or abroad, or from public or private research centers.
L'archive ouverte pluridisciplinaire HAL, est destinée au dépôt et à la diffusion de documents scientifiques de niveau recherche, publiés ou non, émanant des établissements d'enseignement et de recherche français ou étrangers, des laboratoires publics ou privés.

\section{(1)(1) $\$(0)$}

Distributed under a Creative Commons Attribution - NonCommercial - ShareAlikel 4.0 


\title{
Modelling the dynamics of the piano action: is apparent success real?
}

\author{
Anders Thorin ${ }^{1,2 *}$, Xavier Boutillon ${ }^{1}$, José Lozada ${ }^{2}$
}

\begin{abstract}
The kinematics and the dynamics of the piano action mechanism have been much studied in the last 50 years and fairly sophisticated models have been proposed in the last decade. Surprisingly, simple as well as sophisticated models seem to yield very valuable simulations when compared to measurements. We propose here a very simple model, with only 1-degree of freedom, and compare its outcome with force and motion measurements obtained by playing a real piano mechanism. The model, purposely chosen as obviously too simple to be predictive of the dynamics of the grand piano action, appears either as very good or as very bad, depending on which physical quantities are used as the input and output. We discuss the sensitivity of the simulation results to the initial conditions and to noise and the sensitivity of the experimental/simulation comparisons to the chosen dynamical model. It is shown that force-driven simulations with position comparisons, as they are proposed in the literature, do not validate the dynamical models of the piano action. It is suggested that these models be validated with position-driven simulations and force comparisons.
\end{abstract}

\section{Keywords}

piano action, piano key, model validation, multibody dynamics, haptics

${ }^{1}$ Laboratory for Solid Mechanics (LMS), École Polytechnique, F-91128 Palaiseau Cedex, France.

2 CEA, LIST, Sensorial and Ambient Interfaces Laboratory, 91191 Gif-sur-Yvette, France.

* Corresponding author: anders.thorin@polytechnique.edu

\section{Introduction}

The mechanical function of the piano action is to throw the hammer towards the strings. As a human-machine interface, its role is to provide the pianist with a means to perform the following musical task: obtain a given impact velocity of the hammer on the strings at a given instant, with as much as precision as possible. We focus here on the grand piano action but all what is proposed here would apply to the upright piano mechanism.

Piano actions are complex systems mostly resulting from engineering during the 18th and 19th centuries, mostly by trialand-error. The assembly of dozens of pieces is the fruit of a few major inventions (particularly by Cristofori and Érard, for the mechanisms that remained in the 20th century) and many minor refinements. In the resulting sophisticated design, it is not any more obvious to distinguish what are the features due to engineering-economy, ease of manufacturing in given historical conditions, necessity of a silent motion, ease of repair and adjustment, etc.- - and those imposed by piano playing requirements: ease and precision of control, compliance with the playing tradition. Actions that are built for digital pianos (sound synthesisers) can be seen as tentatively complying with the latter group of requirements by means of markedly different engineering solutions. Although constantly improving over years, it is interesting to notice that the results are not yet judged as entirely convincing.

We focus here on the dynamics of the piano action-the forcemotion relationship - as seen from (or felt at) the finger-end of the key. In this paper, the piano action is considered either subject to a given force or to a given motion, which would be imposed by an operator. As of today, the physical quantity controlled by the pianist during the keystroke (or before) in order to perform the musical task has not been identified. In reality, the dynamics of the piano action is coupled to that of the finger/hand/arm/. . . musculoskeletal system which is coupled itself to a neurological system of efferent and afferent nerves. A vast literature is available on various questions pertaining to the pianist control, involving sensory-motor questions as well as the dynamics of the pianist limbs and fingers (see for example [1, 2, 3]). This complex question is not analysed here.
Since the 60's, many dynamical or mechanical models of the piano action have been proposed, each of their authors more or less claiming that it emulates successfully the kinematics (usually the angular positions of the key and hammer) or the dynamics of the mechanism as seen at the end of the key. These claims are usually supported by comparisons between experimental measurements and numerical results issued by the model. The experimental results are generally obtained by imposing a force (constant or varying in time) on the key, by measuring this force, the resulting motion of the key, that of the hammer and, sometimes, of other pieces. Since it is not known whether the control by the pianist is more of a force- or a motion-nature, the choice of given force-profiles or motion-profiles for controlling the dynamics in experiments and simulations may appear as more or less arbitrary, and irrelevant for validating a given model. This paper aims at demonstrating that this is not so. To this end, we analyse the predictions of very simple models of the mechanism, with only one degree-of-freedom.

A few elementary models of the piano action or of some of its parts have been proposed in the first half of the 20th century. In 1965, a frictionless model with superimposed masses is proposed by Dijksterhuis [4]. Oledzki [5] studied a model where two masses (one for the hammer and one for all the other parts) were connected by a spring, representing the internal flexibility of the action. Gillespie and Cutkosky [6] presented a model with four bodies (key, whippen, jack and hammer) where damping, compliances and friction were neglected. In [7], one model is considered for each set of kinematic constraints. A unidimensional model was exposed by Mori [8], who applied forces to the key with calibrated weights. Another model was proposed by Hayashi et al. [9], consisting in a 2-DOF model with a free mass representing the hammer. Contrary to all the other simulations in the literature which are driven by forces, Hayashi's are driven either by a constant velocity, or by a constant acceleration. However, forces are not considered in this paper. A 2-DOF model is also proposed by Oboe [10]. The key and the hammer are modelled, neglecting friction, but the escapement is not considered. In 1995, Van den Berghe et al. [11] considered a 3-DOF model 
where the whippen-lever-jack assembly is rigid. The escapement is therefore not modelled either. The kinematics in response to a force input is discussed.

More complex models appear in the late 90s. The repetition lever is taken into account in [12]. A complete model (5-DOF, the damper is ignored), with measured parameters, is proposed by Hirschkorn [13]. Links presents a similar model [14]. Lozada [15] gives a different model with all the values of its parameters It also includes the first attempt of driving the simulations with a position, without success. Recently, Bokiau et al. [16] have also proposed a rather sophisticated model. Force-driven simulations yield the motion of various pieces.

Except those of [9] and [15], all the simulations were driven with a force input (sometimes, the applied force is constant or idealised), and the resulting kinematics was observed.

In this study an amateur pianist played a real key mechanism at three different dynamical levels, with the finger directly acting on the key and resting on the key before the beginning of the motion. The corresponding motion of the key and the force acting on it is recorded (Section 2). We then consider very simple models, so simple that they can hardly be considered as valid (Section 3 ). Their parameters are derived from static measurements on the real mechanism and from measurements on separate pieces that have been taken apart. The models predict the resulting motion for a given force exerted at the end of the key. When driven by an imposed motion, they can predict the reacting force as well. Comparisons are made between the measured and the predicted motions in the first case, and between the measured force and the predicted force in the other one (Section 4). The matching between the former appears to be much better than between the latter, motivating the discussion in Section 5.

\section{Experiments}

The experiments are performed on a single piano key mechanism (Figure 1) manufactured by the Renner factory for demonstration purposes but similar to the mechanisms in use in grand pianos, particularly with respect to its regulation possibilities. The action has been carefully adjusted by a professional piano technician in line with the standards observed in a piano keyboard. We stabilised the provided sample action by screwing it to a thick metal plate and fixing it solidly to a heavy support. This action is presumed to be representative enough of the grand piano action in general, for the purpose of this paper.

Compared to normal playing, a few modifications have been introduced. The damper has been removed (which corresponds to a "normal" playing condition with the right pedal engaged). It appeared that some experiment-simulation comparisons are sensitive to the precise initial position of the key. Such small variations in initial key position occur normally due to the felt supporting the key at rest (left end of the key in Figure 1). As this condition is not critical to the questions addressed in the study the felt was replaced by a rigid support.

We consider four phases during a keystroke: the first phase of the motion ends when the hammer escapes, the second phase when it is checked, the third phase lasts until the key is released and the last phase when the key comes back to rest. For a detailed description of the timing of the piano action, see [17].

The position of the key is measured by laser-sensors (Keyence LB12, with LB72 conditioning amplifier). The response time of this measuring system was $0.15 \mathrm{~ms}$ and the resolution was $50 \mu \mathrm{m}$. The error due to nonlinearity was less than $100 \mu \mathrm{m}$. Two measurements were done: at the end of the key and approximately mid-way between the finger-end and its rotation centre. When multiplied by the correct ratio of lengths, the high degree of similarity between the two signals was a good indication that the key motion can be considered as a rotation, within the precision required by the conclusions of this paper.

The position of the end of the key, denoted by $y$, is related to the angular position of the key $\theta$ by $y=\theta L$, where $L$ is the length between the finger-end and the rotation centre (see Figure 4). Two particular positions of the key ( $y=y_{\mathrm{e}}$ and $y=$ $y_{\mathrm{p}}$ or equivalently $\theta=\theta_{\mathrm{e}}$ and $\theta=\theta_{\mathrm{p}}$ ) and the corresponding times $\left(t_{\mathrm{e}}\right.$ and $\left.t_{\mathrm{p}}\right)$ at which they are measured are reported in the figures of this article by black dashed-lines and black continuous lines, respectively. The angular position $\theta_{\mathrm{e}} \approx 0.035 \mathrm{rad}$ has been evaluated in a quasi-static test as the angular position of the key when the jack meets the let-off button. When playing, this position corresponds closely to escapement but not exactly since the felts are compressed, depending on how the key has been played. In fact, escapement occurs slightly after (by a variable margin) $\theta(t)$ reaches $\theta_{\mathrm{e}}$. For the sake of brevity in formulation, this slight difference is ignored in the rest of the article. The angular position $\theta_{\mathrm{p}} \approx 0.040 \mathrm{rad}$ corresponds to the key meeting the front rail punching and has also been evaluated in a quasistatic test.

The minimum force initiating down-motion and the maximum force still allowing up-motion from bottom position have been estimated with the standard procedure (adding and removing small masses at the end of the key). They are respectively $F_{\text {down }} \approx 0.70 \mathrm{~N}$ and $F_{\text {up }} \approx 0.38 \mathrm{~N}$, both exceeding by about $0.15 \mathrm{~N}$ the values normally adjusted by technicians.

The key acceleration is measured by a light $(0.4 \mathrm{~g})$ accelerometer (Endevco 2250A-10 with B\&K Nexus measurement amplifier). The bandwidth of the amplifier was chosen as $[0.1 \mathrm{~Hz}, 3 \mathrm{kHz}]$. For practical reasons, the accelerometer was glued approximately mid-way between the end and the rotation centre of the key. A separate experiment performed on forte keystrokes with two accelerometers (one half-way, one at the end of the key), proved that the accelerations at these two points, normalised by their distance to the rotation centre, are nearly undistinguishable until the key reaches the front rail punching. It follows that the acceleration due to translation (slight compression of the pivot support) and the (beam-like) modes of the key seen as a beam can be ignored within the precision required by the conclusions of this paper.

The force exerted on the end of the key is measured with a light-weight (1.2 g) piezoelectric sensor (Kistler 9211, with charge amplifier 5015). The data are sampled at $50 \mathrm{kHz}$ (ADC USB-6211 by National Instruments). The bandwidth of the sensor is larger than the bandwidth of the amplifier, the latter chosen as $[\ll 1 \mathrm{~Hz}, 3 \mathrm{kHz}]$. Data are taken during about $1 \mathrm{~s}$ before the keystroke in order to perform the following post-treatments: the $50 \mathrm{~Hz}$ component is identified in phase and in amplitude on the data before the keystroke and is removed from the entire measurement. The $0 \mathrm{~Hz}$ component is identified and removed in the same way. The resolution of the sensor is $5 \times 10^{-4} \mathrm{~N}$, the errors due to nonlinearity and hysteresis are less than $0.1 \mathrm{~N}$. Combined with the noise evaluated before the keystroke, the measurement uncertainty can be evaluated in the order of magnitude of $0.1 \mathrm{~N}$.

In what follows, the motion of the key is reported at the end of the key (with measured signals multiplied by the appropriate factor) and shifted so that the zero-values correspond to the rest position.

Since the models include viscosity, the key velocity must be 


\begin{tabular}{|c|c|c|c|}
\hline \multicolumn{2}{|c|}{ Nomenclature } & $C_{\mathrm{d} i}$ & Dry friction coefficient of block $i$ \\
\hline \multicolumn{2}{|c|}{ General notations } & $c_{\mathrm{v} i}$ & Viscous friction coefficient of block $i$ \\
\hline$\delta$ & Compression of the felt (length) & $C_{\mathrm{w} i}$ & Torque due to the weight of block $i$ with respect to its \\
\hline$\theta, \dot{\theta}, \ddot{\theta}$ & Angular position, velocity, acceleration of the key & & rotation axis \\
\hline$\theta_{\mathrm{p}}, t_{\mathrm{p}}$ & Value of $\theta, t$ when key meets front rail punching & $J_{i}$ & Moment of inertia of the block $i$ with respect to its rota- \\
\hline & Torque of $F$ & & tion axis \\
\hline$F$ & Force exerted on the key & \multicolumn{2}{|c|}{$l_{12}, l_{21}, l_{23}, l_{32}$ Lengths defined in Figure 4} \\
\hline$F_{\text {down }}$ & Minimum force initiating down-motion from resting posi- & $R_{i j}$ & Reaction force of body $i$ on body $j$ \\
\hline$F_{\mathrm{up}}$ & $\begin{array}{l}\text { tion } \\
\text { Maximum force allowing up-motion from bottom position }\end{array}$ & \multicolumn{2}{|c|}{ Simplistic key (Figure 5) } \\
\hline$k, b, r$ & Parameters of the felt law (3) & $\kappa, \theta_{0}$ & Stiffness, resting angle of rotational spring of $\mathrm{W}-\mathrm{J}-\mathrm{L}$ \\
\hline$L$ & Length between the finger-end and the rotation center & 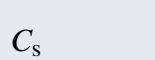 & imposed by the stops \\
\hline $\begin{array}{l}y, \dot{y}, \ddot{y} \\
y_{\mathrm{e}}, \theta_{\mathrm{e}}, t_{\mathrm{e}}\end{array}$ & Position, velocity, acceleration of the end of the key & $C_{\mathrm{w}}, c_{\mathrm{v}}, C_{\mathrm{d}}$ & $\begin{array}{l}\text { Torque due to the weights, viscous friction coefficient, dry } \\
\text { friction coefficient as seen from the key }\end{array}$ \\
\hline \multicolumn{2}{|c|}{ Simplified scheme in three blocks (Figure 4) } & \multicolumn{2}{|c|}{ Abbreviations } \\
\hline \multirow{3}{*}{\multicolumn{2}{|c|}{$\begin{array}{l}\alpha_{21}, \alpha_{32}, \alpha_{31} \text { Geometric dimensionless parameters defined in Eq. (1) } \\
\kappa_{2}, \theta_{2}^{0} \quad \text { Stiffness, resting angle of rotational spring of W-J-L } \\
\theta_{i} \quad \text { Angular position of block } i\end{array}$}} & $\mathrm{BH}$ & "Blockec \\
\hline & & DOF & Degree of freedom \\
\hline & & SK & "Simplistic key" (model) \\
\hline
\end{tabular}

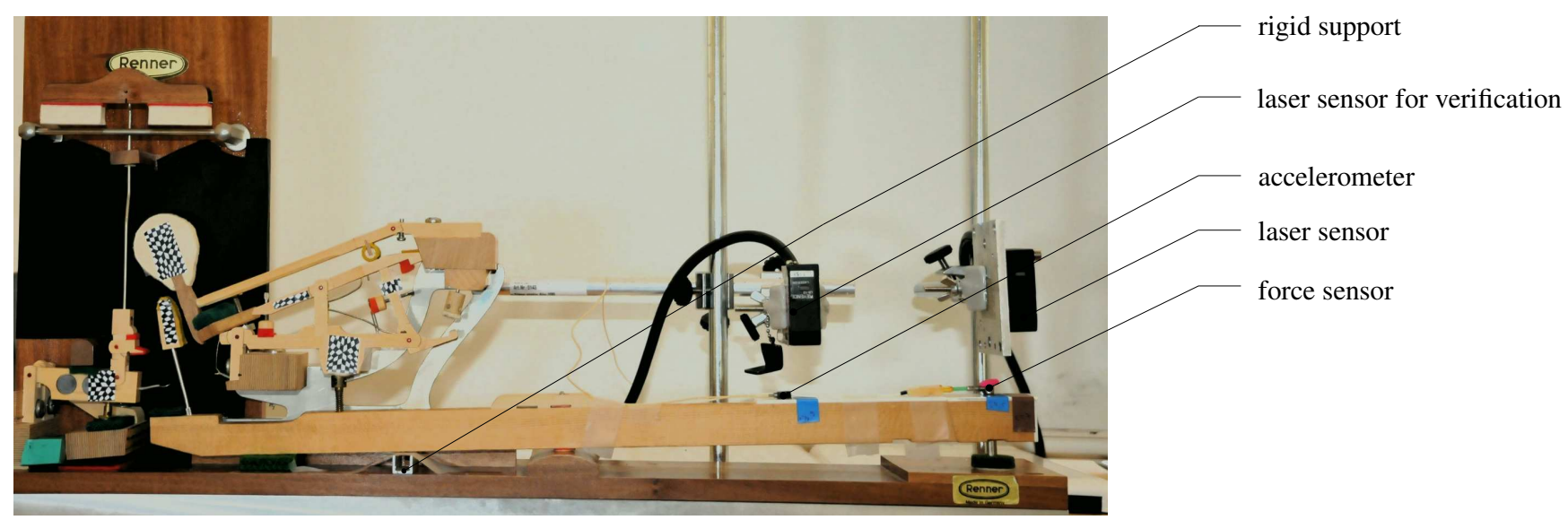

Figure 1. Experimental set-up. The black-and-white patterns were used for experiments which are not reported here.

estimated. The velocity was obtained numerically by two independent algorithms: integration of the acceleration signal (after removal of the average value of the signal at rest) and differentiation of the position signal, using a total-variation regularisation [18] (here: 30 iterations, 200 subiterations, a regularisation parameter of $5 \times 10^{-5}$ and $\varepsilon=10^{-9}$ ). In practice, choosing one or the other estimation of the velocity has very small influence on the simulation results. For practical reasons, we estimated the velocity using the algorithm of total-variation regularisation.

Typical results for mezzo forte playing are displayed in Figure 3. The velocity of the key at the finger's location is positive when the key is pushed down. The same convention applies to the force $F(t)$ on the key.

\section{Simple models}

The mechanism (Figure 2) consists of several quasi-rigid bodies (key, whippen, jack, lever, hammer (the damper has been excluded)) which are coupled together by felts and pivots. A first simplification consists in considering three blocks in the mechanism: the key, the whippen-lever-jack assembly, the hammer (Figure 4). The angular positions of the three blocks shown in Figure 4- $\{$ Key $\}$, \{Whippen-Jack-Lever $\},\{$ Hammer $\}$ - are denoted by $\theta_{1}, \theta_{2}, \theta_{3}$ and their moments of inertia with respect to their rotation axes by $J_{1}, J_{2}, J_{3}$. The sign convention is clockwise for angles and torques and the force $F$ exerted at the end of the key and the motion $y$ of the end of the key are counted positively when the key is pushed down: $y=L \theta_{1}$ and $F=C / L$ $(C$ denotes the torque of $F$ ).

This model does not take into account the compliance between the bodies (key, whippen, etc.) and the contacts with the support (let-off button, drop screw). In line with the spirit of a simple model, it is considered that the variations of the $\theta_{i}$ are small (see Figure 4): the geometrical non-linearities are ignored so that coupling between the parts of the real mechanism does not alter significantly the parameters of the model. Altogether, the mutual dependencies between the $\theta_{i}$ are approximated by geometrical relationships:

$\left\{\begin{array}{l}\theta_{2}=-\frac{l_{12}}{l_{21}} \theta_{1}=-\alpha_{21} \theta_{1} \\ \theta_{3}=-\frac{l_{23}}{l_{32}} \theta_{2}=-\alpha_{32} \theta_{2}=\alpha_{31} \theta_{1}\end{array}\right.$

with the ratio between the hammer angle and the key angle $\alpha_{31}=$ $\alpha_{32} \alpha_{21}$

A more drastically simplified model (Figure 5) consists in a single-degree-of-freedom rotating object with angular position $\theta=\theta_{1}$, referred to as the "simplistic key" (SK) in what follows.

Within the frame of the above approximations, the moment of inertia $J$ of the simplistic key is equivalent to the one of the 


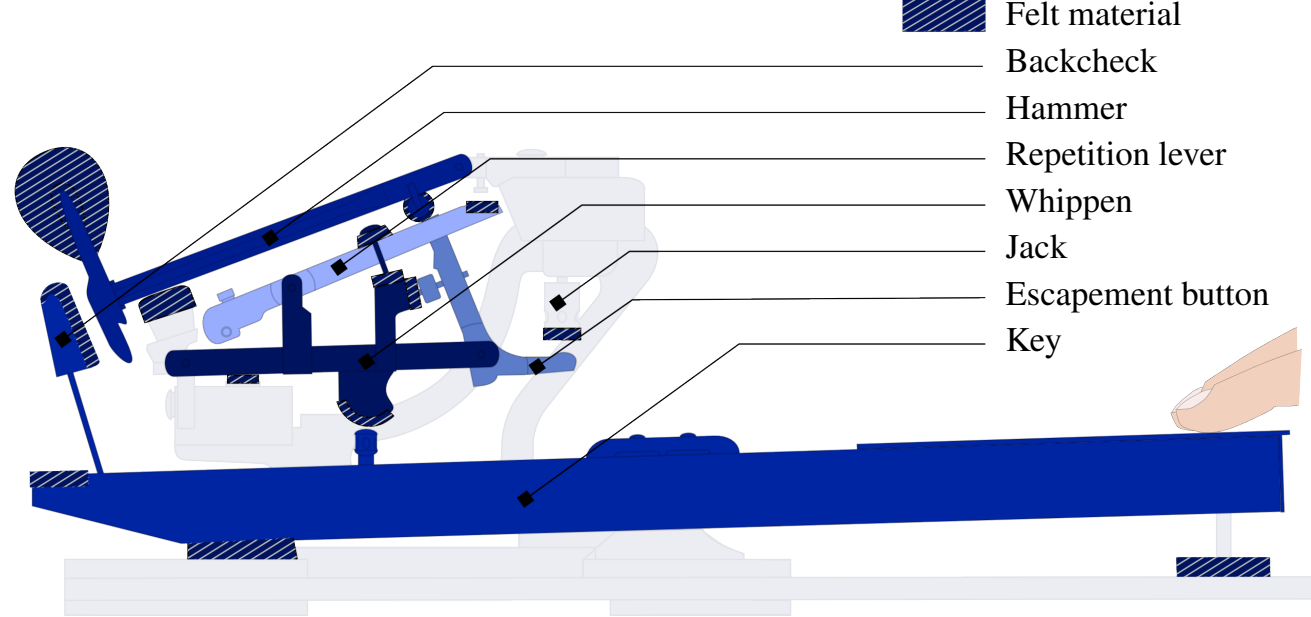

Figure 2. Grand piano action (without damper).
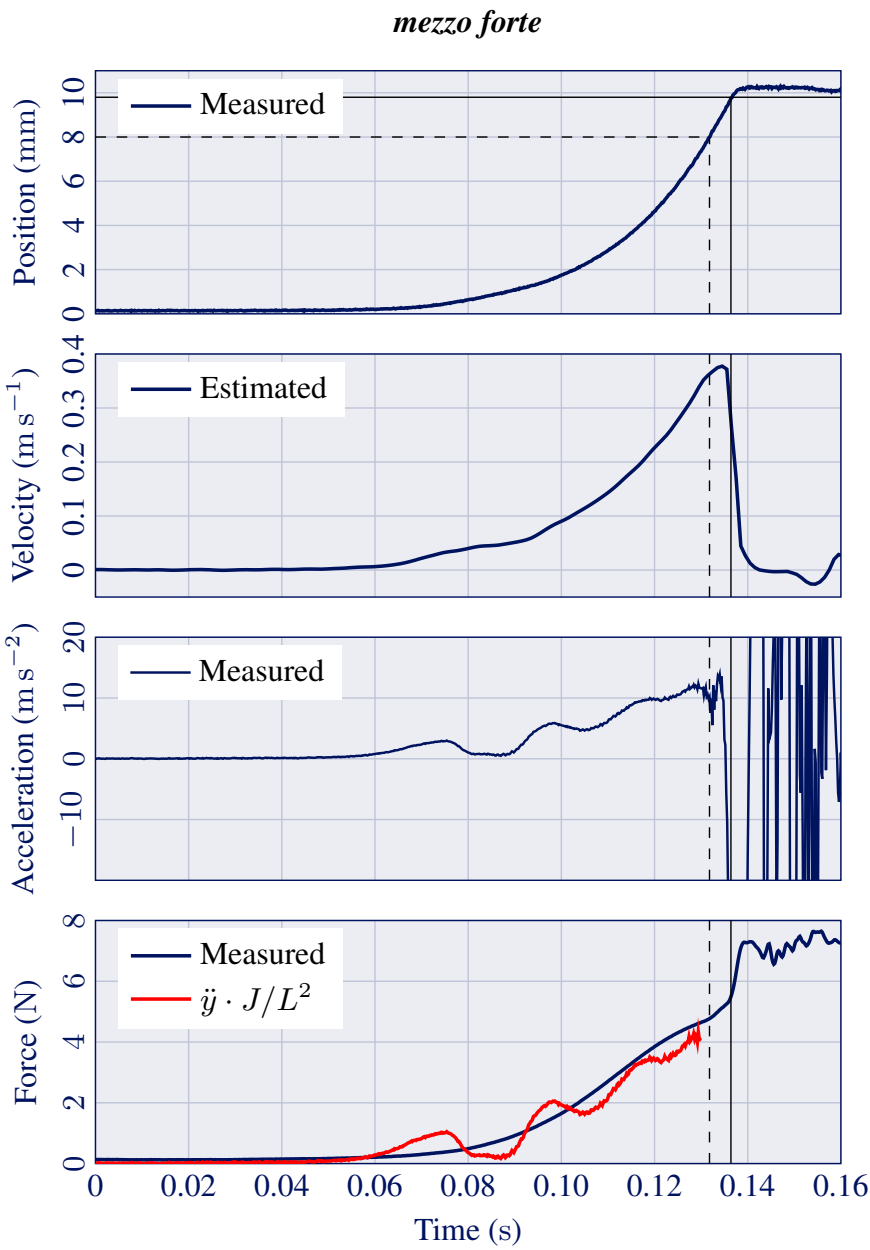

Figure 3. Typical dynamical measurements at the end of the key in a mezzo forte keystroke. The finger is resting on the key before the motion starts. From top to bottom [-]: measured key position $y$, estimated key velocity $\dot{y}$ (see text), measured key acceleration $\ddot{y}$, measured force on the key $F$. The dashed-lines [-- ] and solid lines [-] correspond to $t=t_{\mathrm{e}}$ (escapement of the hammer, see text for additional precisions) and to $t=t_{\mathrm{p}}$ (the key meets the front rail punching) respectively. The acceleration multiplied by $J / L^{2}$ (defined in section 3 ) is reported in the bottom frame ([-]) and discussed in the beginning of section 5. whole mechanism if:

$J=J_{1}+\alpha_{21}^{2} J_{2}+\alpha_{31}^{2} J_{3}$

with the parameters given in Table 1.

For very small key displacements from its rest position, dry friction in the hammer's and the whippen's axes prevents their motion. This lasts at least as long as the force applied to the key is less than $F_{\text {down }}$. Before the force reaches that threshold, we propose further the "blocked hammer" model, yet still very elementary.

The angular momentum of the hammer during acceleration towards the string is several times that of the rest of the mechanism. It follows that the inertia of the whole mechanism differs strongly from that of the simplistic key model when the hammer is dissociated from the rest of the mechanism (a few milliseconds between the escapement and the check of the hammer). Continuation of the model is discussed further in Section 4.

The actions of the torques exerted on the real mechanism are transposed on the simplistic key as follows. Non-permanent torques imposed by the stops limiting the motion of the key are considered and denoted by $C_{\mathrm{s}}$. This torque includes the reaction of the rest support at the inner end of the key which disappears as soon as $\theta(t)>0$ and the reaction of the front rail punching which appears when $\theta(t)>\theta_{\mathrm{p}}$. The compression law $F(L \theta)$ of the front rail punching is that of a felt. Besides the various felt models which have been proposed, we use a phenomenological model including dissipation that has been experimentally validated on that class of felt and geometry [19]:

$F(\delta)=k \delta^{r}+b \delta^{2} \dot{\delta}$

where $\delta$ is the compression of the felt.

After the hammer check, we observe that the hammer looses its kinetic energy much more rapidly than it would with the only dissipation described by Eq. (3). Presumably, the additional source of dissipation is due to friction between the check and the back-check. The key (with the whippen block resting on it) becomes also coupled to the support through the hammer and the back-check felt. Altogether, the value of $b$ has been arbitrarily taken 100 times more than that measured by Brenon in [19]. The values of $k, b$ and $r$ are given in Table 2 .

Permanent torques independent of the key motion include the action of the pianist $C(t)(C(t)=F(t) L)$ and the torques $C_{\mathrm{w} i}$ due to the weights of each different part $i$ of the mechanism. Their 


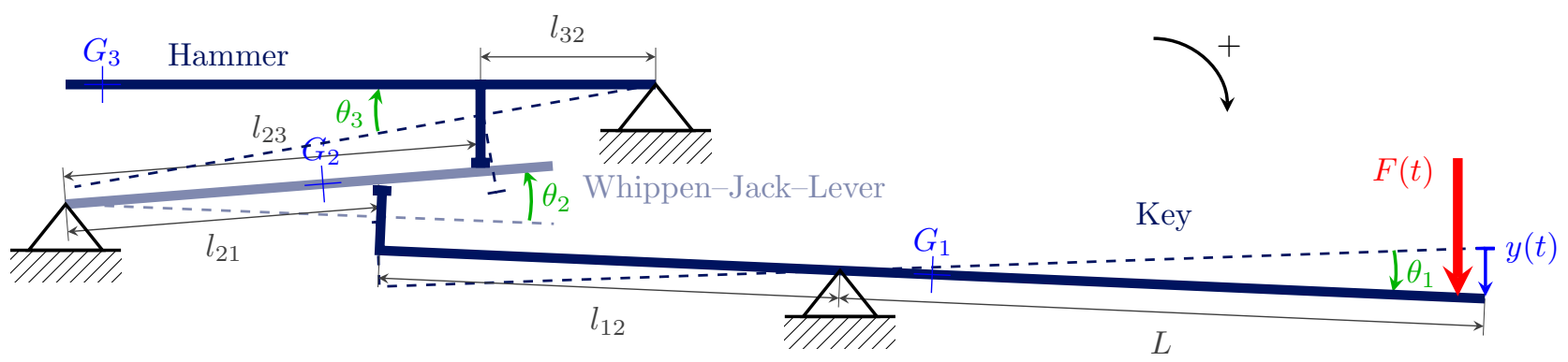

Figure 4. Simplified scheme of the grand piano action in three blocks: Key, Whippen-Jack-Lever, Hammer. The damper has been excluded. The position $\theta_{i}$ of each block is defined as positive when the key is pushed downwards, from 0 at rest. With a clockwise convention for angles (and torques), $\theta_{1}$ and $\theta_{3}$ are positive when they leave their rest position. For the sake of clarity, the reaction forces $R_{i j}$ at the contact points between solids are not represented.

\begin{tabular}{cccccccc}
\hline$i$ & $J_{i}\left(\mathrm{~kg} \mathrm{~m}^{2}\right)$ & $C_{\mathrm{w} i}(\mathrm{Nm})$ & $C_{\mathrm{d} i}(\mathrm{Nm})$ & $c_{\mathrm{v} i}(\mathrm{Nm} \mathrm{s})$ & Spring & Geometry $(\mathrm{m})$ & $\alpha$ \\
1 & $3.36 \times 10^{-3}$ & 0.0155 & 0.012 & 0.022 & & $l_{12}=0.129$ & \\
& & & & & & & \\
& & & & & & & \\
& & & & & & & \\
& $3.97 \times 10^{-4}$ & 0.0103 & & $4.93 \times 10^{-5}$ & $\kappa_{2}=0.087 \mathrm{~N} \mathrm{~m}$, & $l_{21}=0.060$ & $\alpha_{21}=2.15$ \\
& & & & $\theta_{2}^{0}=0.42 \mathrm{rad}$ & $l_{23}=0.080$ & \\
& & & & & & $l_{32}=0.017$ & $\alpha_{31}=10.1$ \\
\hline
\end{tabular}

Table 1. Parameter values of the grand piano action used in experiments according to [15] or measured by us.

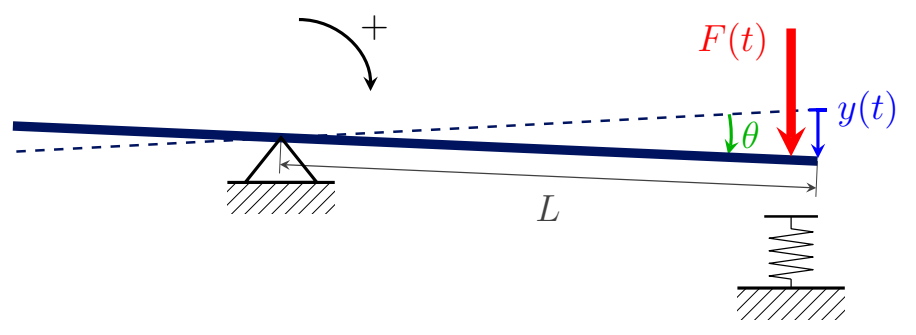

Figure 5. Simple model of the piano action: the simplistic key.

effects amount to $C_{\mathrm{w}}$ given by Eq. (4). Permanent and motiondependent torques due to strains at pivots are modelled by viscous and dry friction: $c_{\mathrm{v} i} \dot{\theta}_{i}$ and $C_{\mathrm{d} i} \operatorname{sign}\left(\dot{\theta}_{i}\right)$ respectively, where $c_{\mathrm{v} i}$ and $C_{\mathrm{d} i}$ are the viscous and dry friction coefficient for the pivot of the part $i \in\{1,2,3\}$. As before, their effect is written as $c_{\mathrm{v}} \dot{\theta}$ and $C_{\mathrm{d}} \operatorname{sign}(\dot{\theta})$. In some mechanisms, including ours, a pre-stressed spring is inserted between the support and the whippen. Its effect is modelled by a torque $\kappa\left(\theta-\theta_{0}\right)$. Within the approximation of small angles, the moments of the coupling forces $R_{i j}$ are given by $R_{i j} l_{i j}$ with the fixed lengths $l_{i j}$ represented in Figure 4 . It comes:

$\left\{\begin{aligned} C_{\mathrm{w}} & =C_{\mathrm{w} 1}-\alpha_{21} C_{\mathrm{w} 2}+\alpha_{31} C_{\mathrm{w} 3} \\ c_{\mathrm{v}} & =c_{\mathrm{v} 1}+\alpha_{21}{ }^{2} c_{\mathrm{v} 2}+\alpha_{31}{ }^{2} c_{\mathrm{v} 3} \\ \kappa & =\alpha_{21}{ }^{2} \kappa_{2}\end{aligned}\right.$

The torques which are proportional to the angular position or velocity (viscous friction, spring) are transferred from 1 DOF to another with squared $\alpha$ factors whereas the torques which do not depend on the angular position or velocity (weight, dry friction) are transferred with $\alpha$ factors.

At the very beginning of the key motion, when $F<F_{\text {down }}$, the force exerted by the key on the whippen is too low to completely overcome the dry friction in the axes of the whippenlever-jack and of the hammer blocks. In consequence, there is a phase where the key moves while the hammer does not: $C_{\mathrm{d} 1} / L<F(t)<F_{\text {down }}$. The hammer behaves as if it was fixed to the support: "blocked hammer" (BH) model. Experimentally, we observed that this model seems to be valid slightly longer than when the force reaches $F_{\text {down }}$. Presumably, this is due to the compression of the small felt below the centre of rotation of the key (in Figure 1 see the small red felt between the middle of the key and the piece of wood supporting it). By various inspections of the motions of the different pieces (position tracking, not reported here), it was found that the dynamics was distinctively changing when $\theta L$ reaches $\approx 0.8 \mathrm{~mm}$. The motion of the key is limited by the compression of coupling felts and springs (in the whippen cushion, etc.). For the sake of simplicity, we model them as one linear equivalent spring. Its stiffness $K$ is estimated as the average ratio between $F_{\text {meas }}$ and $y_{\text {meas }}$ for the three different dynamics considered here.

The dynamical equation of the $\mathrm{BH}$ model is:

$J_{1} \ddot{\theta}+c_{\mathrm{v} 1} \dot{\theta}+C_{\mathrm{d} 1} \operatorname{sign}(\dot{\theta})+K \theta-C_{\mathrm{w} 1}=C(t)$

which is valid for $F_{\text {down }} L \geqslant C(t) \geqslant 0$.

The dynamical equation of the SK model is:

$$
\begin{aligned}
& J \ddot{\theta}+c_{\mathrm{v}} \dot{\theta}+C_{\mathrm{d}} \operatorname{sign}(\dot{\theta})+\kappa\left(\theta-\theta_{0}\right) \\
&-C_{\mathrm{w}}+C_{\mathrm{s}}(\theta, \dot{\theta})=C(t)
\end{aligned}
$$

which is valid for $F_{\text {down }} L \leqslant C(t)$.

The (1-DOF) $\mathrm{BH}$ model has the same structure as the SK model (Figure 5) and differs from it in only two ways: the unilateral constraint $C_{\mathrm{s}}$ which appears when the key hits the front rail punching can be ignored in the initial phase; the values of the parameters are different. The temporal link between the simulations of the two models takes advantage of this property (Section 4).

The parameters of the model can be estimated by means of a few experiments and measurements. The parameters of the right-hand sides of Eq. (2) and Eq. (4) have been estimated by measurements on the separate elements, as described in [15]. 
Their values are given in Table 1 . The other values of the parameters of Eqs. (5) and (6) are given in Table 2. A second estimation of $C_{\mathrm{w}}$ and the estimation of $C_{\mathrm{d}}$ are given by the static test described in Section 2:

$$
\begin{aligned}
\left|C_{\mathrm{w}}\right| & =L \frac{F_{\text {down }}+F_{\text {up }}}{2} \\
C_{\mathrm{d}} & =L \frac{F_{\text {down }}-F_{\text {up }}}{2}
\end{aligned}
$$

The moment of inertia $J$ as defined by Eq. (2) has been evaluated as follows: direct measurements of the period of simple pendulums made of the hammer and the whippen (with an experimental determination of their centre of mass) and calculation according to the mass and shape of the key on the other hand (see [15] for more details). The uncertainty on the value of $J$ is estimated around $5 \%$.

\begin{tabular}{rl}
\hline Parameter & Numerical value \\
$J$ & $0.0221 \mathrm{~kg} \mathrm{~m}^{2}$ \\
$C_{\mathrm{W}}$ & $-0.138 \mathrm{~N} \mathrm{~m}$ \\
& $\left(\right.$ Eq. $(7)$ yields $\left.C_{\mathrm{w}}=0.132 \mathrm{~N} \mathrm{~m}\right)$ \\
$C_{\mathrm{d}}$ & $0.039 \mathrm{~N} \mathrm{~m}$ \\
$c_{\mathrm{V}}$ & $0.0273 \mathrm{~N} \mathrm{~m} \mathrm{~s}^{-1}$ \\
$\kappa$ & $0.4 \mathrm{~N} \mathrm{~m}$ \\
$\theta_{0}$ & $0.1947 \mathrm{rad}$ \\
$\theta_{\mathrm{e}}$ & $0.0343 \mathrm{rad}$ \\
$\theta_{\mathrm{p}}$ & $0.0397 \mathrm{rad}$ \\
$k$ & $1.6 \times 10^{-10} \mathrm{SI} \mathrm{unit}$ \\
$b$ & $2 \times 10^{9} \mathrm{~N} \mathrm{~s} \mathrm{~m}^{-3}$ \\
$r$ & 2.7 \\
$K$ & $60 \mathrm{~N} \mathrm{~m}$ \\
\hline
\end{tabular}

Table 2. Parameters of the SK and BH models.

\section{Simulations}

This part presents simulations of the position of the key in response to given forces (force-driven simulations) and conversely, the reaction of the key to a prescribed motion at its end (motiondriven simulations).

As mentioned in Section 3, the inertial aspect of the model is invalid between the escapement and the check of the hammer. However, we chose to continue the simulation all along.

For a given force profile (here: $F_{\text {meas }}(t)$ ), the angular position of the key $\theta_{\text {simul }}(t)$ (and the displacement $y_{\text {simul }}=L \theta$ of its end) has been obtained by solving numerically Eq. (5) and Eq. (6) with $C(t)=F_{\text {meas }}(t) L$. The numerical integration has been done by the NDSolve function of Mathematica ${ }^{\circledR}$, using an Adams method with a maximum step limit of 30000 .

The results of these force-driven simulations are presented in Figure 6 for three different keystrokes: piano, mezzo forte and forte. The force inputs are represented by the blue dotted lines in Figure 7. For the mezzo forte keystroke, the force input is the same as shown in Figure 3.

The red dash-dotted line in Figure 6 represents the result of the simulation of Eq. (6) (SK model) with initial conditions given by the observation of $\theta$ and $\dot{\theta}$ at $t$ corresponding to $F(t)=F_{\text {down }}$. The drift that can be observed in these simulations is discussed in Section 5.
The black dashed line in Figure 6 represents the result of the simulation of Eq. (5) (BH model) with initial conditions at rest.

Linking in time the simulation of the $\mathrm{BH}$ model to that of the SK model (solid thin red line) when $F(t)=F_{\text {down }}$ was done by linear interpolation of the momentum of inertia $\tilde{J}(\theta)$ and the momentum of weights $\tilde{C}_{\mathrm{w}}(\theta)$ from their values in the first phase to their values in the second phase. This is possible for the reason given above, that the two models have the same structure.
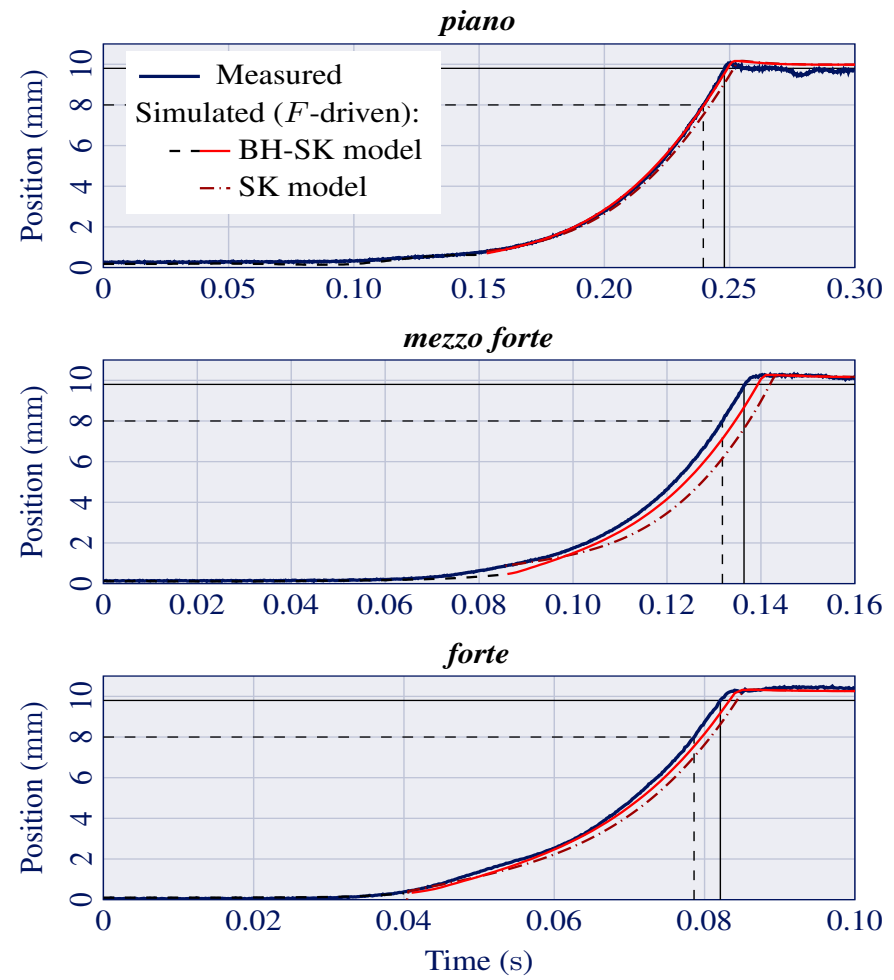

Figure 6. Position y of the end of the key during various strokes (note that the stroke duration is shorter at higher dynamical levels). Simulated positions correspond to measured driving forces represented in Figure 7. [-] Measured position. [- - ] Simulated position according to Eq. (5) (BH model) starting at rest. [-] Simulated position of the SK model linked in time with the BH model by linear interpolation of the parameters (see text). [---] Simulated position according to Eq. (6) (SK model) starting with initial conditions taken in experimental data (see text). Vertical and horizontal lines: see caption of Figure 3.

Motion-driven simulations yield $F_{\text {simul }}(t)=C(t) / L$, the opposite of the reaction force exerted by the key for a prescribed motion $\theta(t)$ (here, $\theta(t)=y_{\text {meas }} / L$ ). According to Eq. (5) and Eq. (6), such simulations are straightforward, once the position and the acceleration of the key have been measured and the velocity has been estimated (see Section 2). The results are presented in Figure 7 for the same keystrokes as in Figure 6. The position, velocity and acceleration inputs are represented in Figures 6, 8 and 9 respectively. For the mezzo forte keystroke, the motion inputs are the same as shown in Figure 3.

In Figure 7, the black dashed line represents the result of the simulation of Eq. (5) (BH model) up to the moment when $F(t)=F_{\text {down }}$. The result of the simulation of Eq. (6) (SK model) is represented by the red dash-dotted line represents before this moment and by a plain thin red line afterwards. 

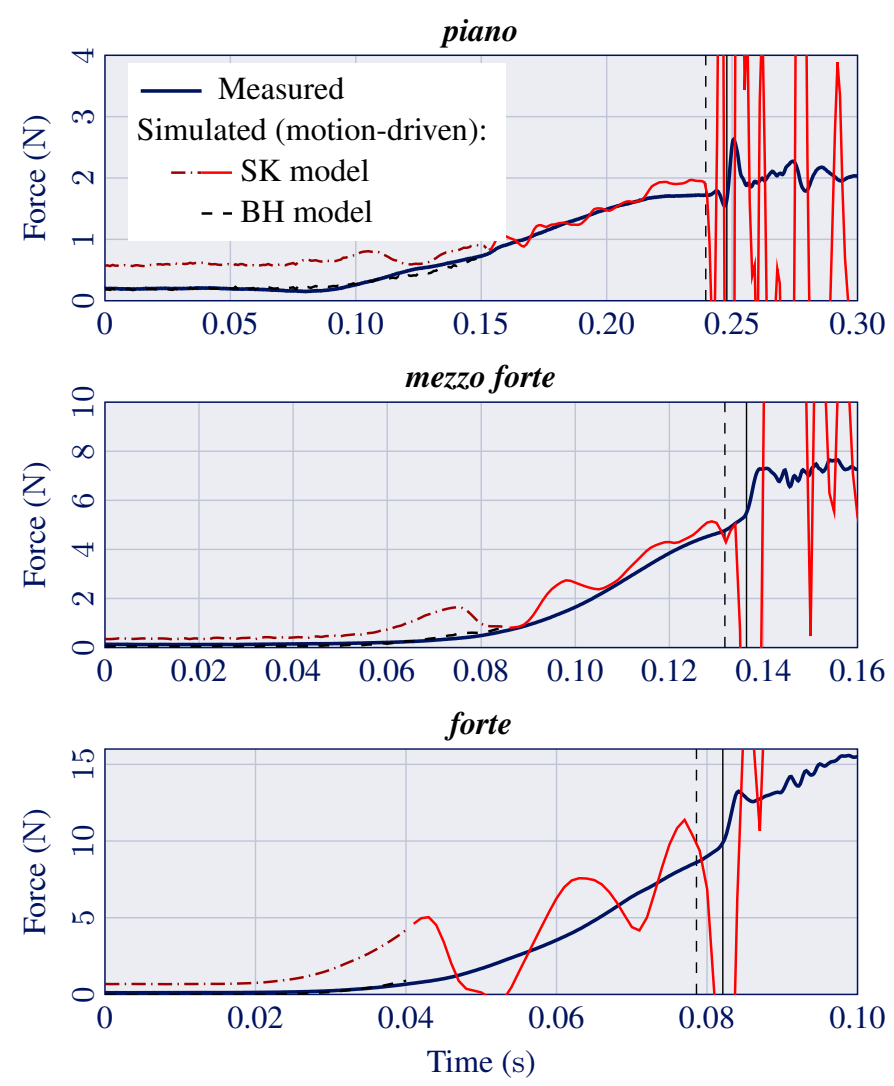

Figure 7. Force $F$ applied on the end of the key during various strokes when driven by a measured key motion profile at three dynamic levels. [-] Measured force. [--] Simulated force according to Eq. (5) (BH model). [--] and [-] Simulated position according to Eq. (6) (SK model), respectively before and after when $F(t)=F_{\text {down. }}$ Vertical lines: see caption of Figure 3.

\section{Discussion}

After escapement, the pianist has no control on the hammer. Furthermore, observing how pianists test and feel a keyboard makes us think that the haptic feedback until escapement (included) is of prime interest to them. Therefore, the discussion is primarily, although not exclusively, focused on this phase of the motion.

A first and basic finding can be deduced from the experimental observations reported in Figure 3. An estimation of the inertial part $\left(J / L^{2}\right) \ddot{y}$ of the force $F$ is represented in the bottom frame, showing that the dynamics of the mechanism is dominated before escapement by the inertia of its pieces, taken as a whole. The other-than-inertial dynamical effects due the internal degrees of freedom, the various stops that are met or left by the pieces, etc. appear as time variations of the difference $F(t)-\ddot{y}(t) J / L^{2}$. The corresponding wiggles can be easily distinguished in the bottom frame of Figure 3, even though the motion (top frame) is quite smooth. Although not surprising, this elementary observation has important implications with regard to the main point raised in the introduction: in order to validate a dynamical model, should the dynamics be examined as producing a force in response to an imposed displacement or vice-versa?

Since the dynamics of this particular mechanism is dominated by inertia until escapement, it follows that the acceleration is roughly as smooth as the force, possibly displaying some wiggles. When imposing a force and looking at the angular position of the key or, equivalently, at the displacement of the end of the key, these potential wiggles in the acceleration are heavily filtered by the double time-integration. It follows that the differences
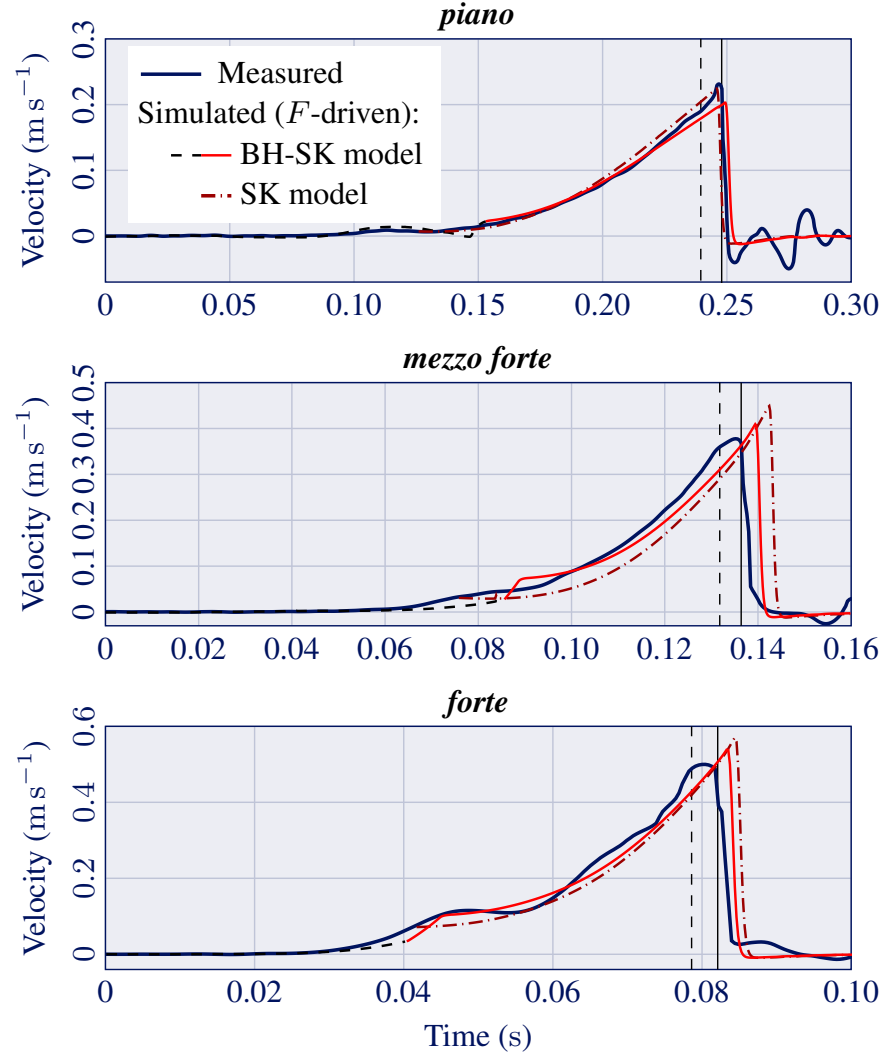

Figure 8. Estimated velocity $\dot{y}$ of the end of the key during various strokes (estimation procedure: see end of Section 2). Same keystrokes and conventions as in Figure 6.

between inertia and the complete dynamics of the system becomes hard, if not impossible, to distinguish. In other words, any model, provided that it is inertia-dominated, is likely to appear as very good when checking its validity by means of comparisons between motion-results obtained in force-driven simulations and tests, before escapement. This lack of sensitivity of the results to the model is represented by a " 0 " in upper left cell in Table 3.

\begin{tabular}{rl|ccc}
\hline Input & Output & Model & Initial conditions & Noise \\
$F$ & $y$ & 0 & + & 0 \\
$\ddot{y}$ and $y$ & $F$ & + & 0 & 0 \\
$y$ & $F$ & + & 0 & + \\
$\ddot{y}$ & $F$ & + & + & 0 \\
$F$ & $\dot{y}$ & 0 & + & 0 \\
$F$ & $\ddot{y}$ & $(+)$ & + & + \\
\hline
\end{tabular}

Table 3. Sensitivity of the results of the simulation, driven as indicated in the first line, to the elements indicated in the first column. "+" means "sensitive". "0" means not or little sensitive.

Bearing that in mind, we analyse now the diagrams in Figure 6, where the "blocked hammer" (BH) and "simplistic key" (SK) models are ruled by a given force and yield a certain motion (solid red line). Our first remark pertains to the sensitivity of the SK model to initial conditions. Simulations based on the SK model alone are represented by a dashed line. They are run with initial conditions that are given by the experimental data: position and velocity for an arbitrary force level, slightly exceeding $F_{\text {down }}$. The corresponding curves display a drift compared to those issued from the succession of the $\mathrm{BH}$ and SK models. This denotes a high-sensitivity of the SK-model to the initial 

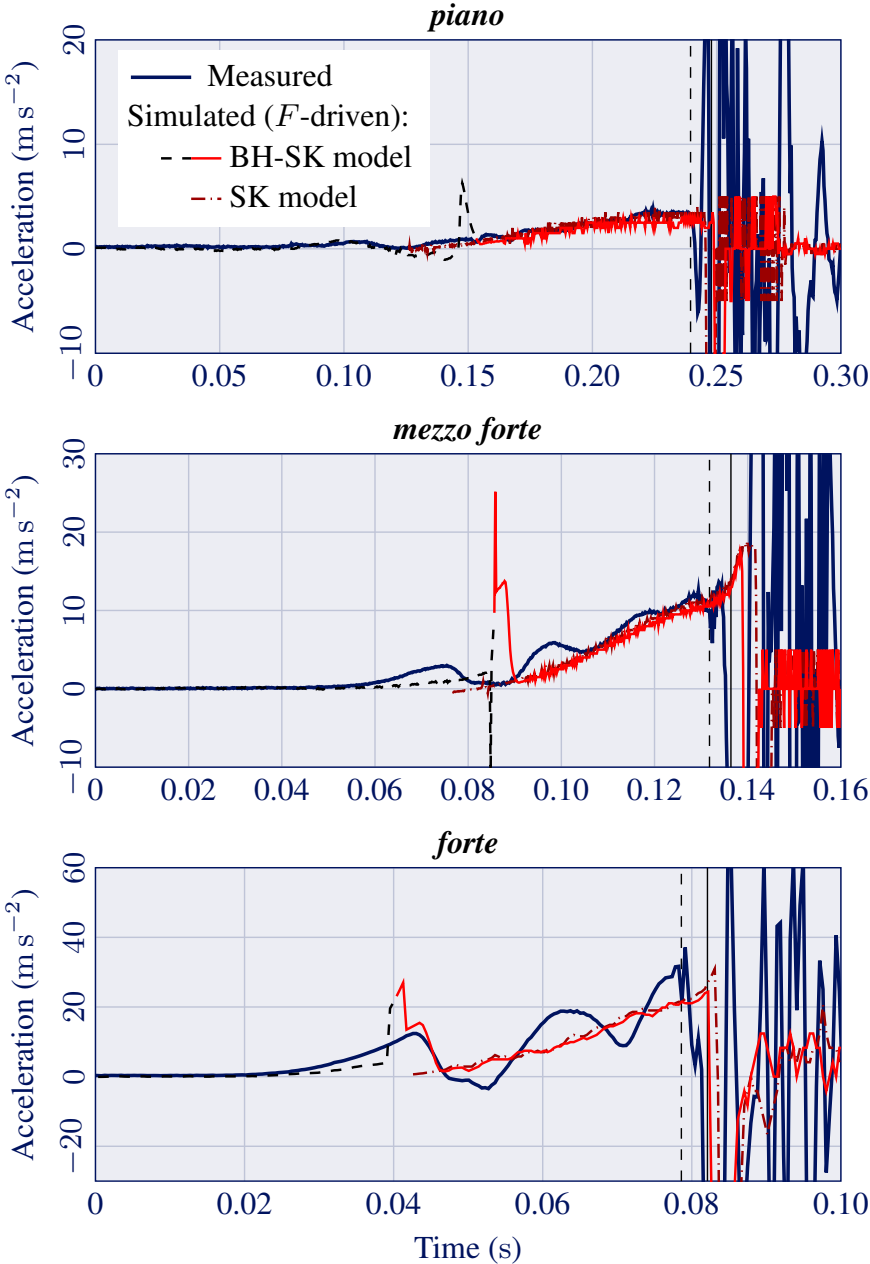

Figure 9. Acceleration $\ddot{y}$ of the end of the key during various strokes. Same keystrokes and conventions as in Figure 6.

conditions, more precisely, the initial velocity, consistent with the fact that the dynamics is dominated by inertia. This sensitivity is represented by a "+" in the second cell of the left-most column in Table 3. The experiment/simulation comparisons in Figure 6 seem to fully validate the "blocked hammer" model succeeded by the "simplistic key" model, including after escapement and the check of the hammer. Although these are obviously very crude models, this apparent match is consistent with the above remark that any model seems correct under the circumstances and restrictions described above (upper left " 0 " in Table 3).

Simulations with the same model driven by the motion data (instead of the force data) issued from the same experimental tests, yield force results that look, by contrast, very different from their experimental counterparts. The experimental/simulation comparisons presented in Figure 7 cannot be considered at all as a validation of the "simplistic key" model. Only a more elaborate model could yield simulation results that would better match the observations. This sensitivity to the model is summarised by the "+" in the second column of the first line in Table 3. The oscillations that appear in the simulations of the force in the SK model, scaling with the dynamic level, are consistent with the observed accelerations (see Figure 9). Although not of essential interest, it is worth noticing that at the very beginning of the motion, the BH model seems to predict correctly the kinematics (force-driven simulations) as well as the dynamics (motion-driven simulations). The $\mathrm{BH}$ model thus appears as valid during the very initial part of the motion, unlike the SK model. Therefore, one should not be surprised that, in the bottom frame of Figure 7, the BH model does not link smoothly with the SK model.

The results reported here have been obtained by using both position and acceleration experimental data. If only position data are available, the velocity and the acceleration must be calculated by successive time-derivations, before escapement. The result is known to be very sensitive to noise in the position data. Conversely, using acceleration-data only requires successive timeintegrations, generating drifts which denote a high-sensitivity to the determination of initial conditions. For a synthesis of these remarks, see the corresponding cells in Table 3.

In the literature on piano action modelling, many authors chose to report their results in terms of the kinematics of the hammer. We did not represent the motion of the hammer for the following reason. In the $\mathrm{BH}$ model, the angular position of the hammer is zero; in the SK model and up to escapement, (see the geometrical relationships Eq. (1) between the angular positions of the pieces) it is proportional to the shifted angular position of the key (the shift corresponding to the angular position of the key when the hammer just begins to move).

The first set of comparisons presented by Oboe [10] (paper's Figure 18) is purely kinematical. His second set (paper's Figure 19) presents key and hammer motions in response to a force applied to a 2-DOF model. Van den Berghe [11] presents a $3-$ DOF model. Again, the resulting key's and hammer's motions in response to a force look very similar to measurements. Using different 2-DOF models for different kinematic constraints, Gillespie [20] also compares the calculated and measured key's displacements (paper's Figure 9). Somme rubber has been added between the bodies in order to regularise the behaviour of the system. Hirschkorn \& al. [13] use a 5-DOF model to compute the hammer's and key's positions for a given force input, in piano and in forte (paper's Figures 12 to 15). The authors deduce from the similarities with measurements that the model predicts the behaviour of the piano action with reasonable accuracy. The kinematics of the bodies are calculated by Links [14] with a 5-DOF model for a force input (paper's Figures 5.3 to 5.6) and match well the measurements.

All these authors (a) compare simulated kinematics in response to a force-profile and (b) find a good agreement with measurements. According to the previous remarks, (b) is quite understandable. However, it would be surprising that so significantly different models could be valid up to the same precision. Our first and main conclusion is that looking at the resulting motion of force-driven simulations cannot discriminate between good and bad models (provided they are inertia-dominated before escapement) and thus is not appropriate as a validation method. Moreover, the sensitivity of the simulated position of the key (or of the hammer) to the initial conditions is another reason for ruling out this choice of simulating the dynamics of a piano action by means of a force input. We recall here that this sensitivity is due to the dominance of inertia in the dynamics, not to the model itself.

Some events are very important for the pianist's feeling and control such as the jack/let-off button contact (dashed vertical lines in the diagrams) or the escapement of the hammer. The model presented here and other models with 1 or 2 DOF do not take them into account, probably making them haptically irrelevant. What matters here is that this irrelevance of the model cannot be seen in results of force-driven simulations. Conversely, after let-off, the measured force becomes totally different from the force calculated by motion-driven simulations of the SK model, 
which is reassuring.

Almost immediately after escapement, the key meets the front rail punching (solid vertical lines in the diagrams), the dynamics of the mechanism is dominated by the corresponding nonlinear spring, with additional high losses representing the effect of the back-check (see Eq. (3) and the physical justification of dissipation). This can be seen in the right part of the top and bottom frames of Figure 3: the position is almost constant and the force on the key has a main constant component with, again, some wiggles. Following the same analysis as above, we can conclude that any model which includes a stop with the appropriate stiffness is likely to appear as very good if one looks at the motion, considered as the output of a dynamical system subject to a smooth force profile.

The rapidly changing forces represented in the bottom frame of Figure 3, or the forces measured in Figure 7 are not suitable inputs for validating a dynamical model since the rapid changes in the force are the dynamics of the piano action: using them as the input of a dynamical model amounts to using the solution of a problem for solving it.

After escapement, the SK model is intuitively less appealing than before escapement: the change in momentum of the hammer is ignored, as is the blocking of the hammer due to the backcheck. However, driving the SK model with the measured forces yields an excellent agreement with the measured displacement (Figure 6). Conversely, tests where (smooth) position profiles are used as inputs yield simulated forces which differ strongly from the measurements (Figure 7). The previous reasoning on the apparent validity of inertia-dominated models can be transposed, after escapement, to stiffness-dominated models. The conclusion is the same: matching a measured motion to that given by forcedriven simulations cannot prove that a dynamical model is valid.

It has been suggested by one reviewer of this paper that validating a model could be done by comparing simulated velocities and accelerations (force-driven simulations) to their experimental counterparts. Such comparisons, which have never been presented in the literature, are displayed in Figure 8 and Figure 9 for the same piano, mezzo forte and forte keystrokes as in Figure 6. As mentioned above, the $\mathrm{BH}$ model seems to be valid at the very beginning of the motion. At the forte level, it seems that the inertia is underestimated in this model, meaning that the whippen may move together with the key at the beginning of this keystroke. Between the end of the initial phase of the motion (described by the BH model as explained above) and escapement, all velocity diagrams and the piano acceleration diagram suggest (again, but wrongly as shown above) that the SK model is valid. The validity is questionable on the mezzo forte acceleration response and ruled out by the forte acceleration diagram. Unlike the position and the velocity, the acceleration appears as a discriminating criterion, at high dynamical levels of playing only.

At this point, one must recall that controlling the piano nuance is known to be a major challenge in piano playing and therefore, pianists are likely to be fairly sensitive to the dynamics of the action at this level. The fact that acceleration results of the forcedriven simulation at this dynamical level are not model-sensitive is an additional suggestion that the pianist's control is exerted on the kinematics rather than on the force.

As presented earlier in this discussion, these results of forcedriven simulations are sensitive to initial conditions and acceleration results appear as sensitive to noise, hence the completion of Table 3.

\section{Conclusion}

Observations of the dynamics of the piano action show that it is dominated by inertia before escapement and by stiffness after the key has met the front rail punching. By means of simulations of very simple models, we have shown that the comparison between measured positions (of the key or the hammer) and positions given by force-driven simulations cannot validate a dynamical model of the piano action. A comparison between measured and simulated accelerations may be an alternative, provided that a sufficiently high dynamical level is used. A warning must be issued on the fact that pianists are probably sensitive to details of the dynamics in piano playing whereas simulated accelerations are not, at that level. Although they may be subject to noise, comparing measured and simulated forces in response to position-driven simulations appear as preferable. Noise sensitivity disappears when using also acceleration data. A minor conclusion of this paper is that the blocked-hammer model seems to be valid during the very first stage of the key motion. However, the main parameter (stiffness) of this model was adjusted here.

Driving the mechanism (whether experimentally or virtually) with a force profile yields kinematic information. A 1-DOF model is sufficient to account for the kinematics of the key all along. Only one parameter (damping after hammer check) of the 1-DOF model had to be somewhat arbitrarily chosen. Although the bibliography of the late fifteen years presents 2- or more DOF models, a 1-DOF model may also well be sufficient for rendering the hammer kinematics before escapement.

Driving the mechanism with a motion profile and looking at the reaction force reveals the dynamics. The complexity of the internal dynamics is reflected in the rapid wiggles of the measured forces (and acceleration at the forte level). Only a sophisticated model may render the dynamics of the action, possibly one of those which have been published, if it meets the force-comparison test.

\section{References}

[1] K.C. Engel, M. Flanders, J.F. Soechting. "Anticipatory and sequential motor control in piano playing". Experimental brain research 113(2) 1997, 189-199. DOI: $10.1007 / \mathrm{BF} 02450317$.

[2] D. Parlitz, T. Thomas Peschel, E. Altenmüller. “Assessment of dynamic finger forces in pianists: effects of training and expertise". Journal of biomechanics 31(11) 1998, 1063-1067. DOI: 10.1016/S0021-9290(98)00113-4.

[3] B.H. REPP. "Effects of auditory feedback deprivation on expressive piano performance". Music Perception 1999, 409-438.

[4] P.R. Dijksterhuis. "De piano". Nederlandse Akoest. Genootschap 7 1965, 50-65.

[5] A. OleDZKI. "Dynamics of piano mechanisms". Mechanism and Machine Theory 7(4) 1972, 373-385. DOI: $10.1016 / 0094-114 \mathrm{X}(72) 90047-\mathrm{X}$.

[6] B. Gillespie, M. CutKosky. "Dynamical modeling of the grand piano action". Proceedings of the International Computer Music Conference. International Computer Music Association. 1992, 77 77.

[7] B. GILLESPIE. "The virtual piano action: design and implementation". Proceedings of the international Computer Music Conference. International Computer Music Association. 1994, 167167.

[8] T. MORI. "A comparison between upright and grand pianos". Volume 83. Journal of the European Acoustics Association, 1997. 
[9] E. HAYAShI, M. YAMANE, H. MoRI. "Behavior of piano-action in a grand piano. I. Analysis of the motion of the hammer prior to string contact". The Journal of the Acoustical Society of America 105 1999, 3534-3544.

DOI: $10.1121 / 1.424678$.

[10] R. ОвоЕ. "A multi-instrument, force-feedback keyboard". Computer Music Journal 30(3) 2006, 38-52.

DOI: $10.1162 /$ comj . 2006.30.3.38.

[11] G. Van den Berghe, B. De Moor, W. Minten. "Modeling a grand piano key action". Computer Music Journal 19(2) 1995, 1522.

DOI: $10.2307 / 3680597$.

[12] B. Gillespie. "Haptic display of systems with changing kinematic constraints: the virtual piano action". Ph.D. thesis, Stanford University 1996.

[13] M. Hirschkorn, J. McPhee, S. Birkett. "Dynamic modeling and experimental testing of a piano action mechanism". Journal of computational and nonlinear dynamics 1(1) 2006, 47-55. DOI: $10.1115 / 1.1951782$.

[14] H. LINKS. "Modeling of a grand piano action mechanism". Master thesis, Delft University of Technology 2011.
[15] J. LOZADA. "Modélisation, contrôle haptique et nouvelles réalisations de claviers musicaux (Model, haptic control and new realisations of musical keyboards)". Ph.D. thesis, École Polytechnique 2007.

OAI: tel-00280538v1.

[16] B. Bokiau, A. Poncelet, P. Fisette, N. Docquier. "Multibody model of a grand piano action aimed at understanding and demystifying the escapement principle”. The 2nd Joint International Conference on Multibody System Dynamics, 2012.

[17] A. AsKenfelt, E.V. JANSSON. "From touch to string vibrations. I: Timing in the grand piano action". The Journal of the Acoustical Society of America 88 1990, 52-63. DOI: $10.1121 / 1.399933$.

[18] R. CHARTRAND. "Numerical differentiation of noisy, nonsmooth data". ISRN Applied Mathematics 2011.

[19] C. Brenon. "Mécanique de la touche de piano". Master thesis, Université Pierre et Marie Curie 2002.

[20] B. Gillespie, B. Yu, R. Grijalva, S. Awtar. "Characterizing the feel of the piano action". Computer Music Journal 35(1) 2011, 43-57.

DOI: $10.1162 /$ COMJ_a_00039. 